\title{
A JOINT TEST OF FRACTIONAL CYCLIC INTEGRATION AND A LINEAR TIME TREND
}

\author{
L. A. GIL-ALANA* \\ Humboldt Universität zu Berlin, Institut für Statistik und Ökonometrie, Berlin, Germany \\ University of Navarre, Department of Economics, Pamplona, Spain
}

\begin{abstract}
We propose in this article a joint test for testing simultaneously a deterministic trend component and the degree of integration of the cyclical component in a given time series. The test is directly derived from Robinson's (1994) procedure, which is based on the Lagrange Multiplier (LM) principle. Thus, it has standard null and local asymptotic distributions. However, finite-sample critical values of the tests are evaluated and, an empirical application using historical annual data, is also carried out at the end of the article.

KEYWORDS: Fractional cycles; Deterministic trends; Long memory
\end{abstract}

Correspondence author: Luis A. Gil-Alana

Humboldt Universität zu Berlin

Institut für Statistik und Ökonometrie

Spandauer Str. 1

D-10178 Berlin, Germany

Phone: $\quad 00493020931682$

Fax: $\quad 00493020935712$

E-mail: $\quad$ alana@wiwi.hu-berlin.de

\footnotetext{
* Financial support from Macroeconomic Modelling and Policy Analysis in a Changing World Grant No. "L116251013" is gratefully acknowledged. Also, the author also acknowledges financial support from the Deutsche Forschungsgemeinschaft, SFB 373.
} 


\section{INTRODUCTION}

Modelling cycles in macroeconomic time series has been a major focus of attention in recent years. Deterministic cycles were initially proposed but they were shown to be inappropriate for many time series. Stochastic cycles were proposed amongst others by Harvey (1985) and Ahtola and Tiao (1987), and they were generalised to allow for long memory by Gray et al. (1989, 1994). In particular, they considered processes like

$$
\left(1-2 \mu L+L^{2}\right)^{d} x_{t}=u_{t}
$$

where $\mathrm{L}$ is the lag operator $\left(\mathrm{Lx}_{\mathrm{t}}=\mathrm{x}_{\mathrm{t}-1}\right) ; \mathrm{d}$ is a given real number and the periodicity is determined by $\mu$. If $\mathrm{u}_{\mathrm{t}}$ is $\mathrm{I}(0)$, defined in this context as a covariance stationary process with spectral density which is positive and finite at any frequency, $x_{t}$ in (1) follows a cyclic $I(d)$ process. Gray et al. (1989) showed that the polynomial in (1) can be expressed in terms of the Gegenbauer poynomial $C_{j, d}(\mu)$, such that for all $d \neq 0$,

$$
\left(1-2 \mu L+L^{2}\right)^{-d}=\sum_{j=0}^{\infty} C_{j, d}(\mu) L^{j}
$$

where

$$
C_{j, d}(\mu)=\sum_{k=0}^{[j / 2]} \frac{(-1)^{k}(d)_{j-k}(2 \mu)^{j-2 k}}{k !(j-2 k) !} ; \quad(d)_{j}=\frac{\Gamma(d+j)}{\Gamma(d)}
$$

and $\Gamma(\mathrm{x})$ means the gamma function. Gray et al. (1989) showed that $\mathrm{x}_{\mathrm{t}}$ in (1) is stationary if $|\mu|<1$ and $\mathrm{d}<0.50$ or if $|\mu|=1$ and $\mathrm{d}<0.25$. Simulated realizations based on fractional models like (1) can be found in Gray et al. (1989), and an empirical application in GilAlana (2001).

In this article, we propose a testing procedure for testing simultaneously the degree of integration of the cyclical component in a given time series and the need of a linear time trend. The structure of the paper is as follows: Section 2 describes the tests of Robinson (1994) which permit us to test fractional cyclic models like (1). In Section 3, the tests are extended to allow us to test simultaneously the order of integration and a linear time trend. 
Section 4 evaluates finite-sample critical values of the tests described in sections 3 and 4 , conducting Monte Carlo experiments to check the sizes and the power properties of the tests in finite samples. Section 5 contains an empirical application and finally Section 6 concludes.

\section{THE TESTS OF ROBINSON (1994)}

Following the discussions of Bhargava (1986), Schmidt and Phillips (1992) and others of parameterization of unit-root models, Robinson (1994) considers the following regression model,

$$
y_{t}=\beta^{\prime} z_{t}+x_{t}
$$

where $y_{t}$ is the time series we observe; $\beta$ is a $(k x 1)$ vector of unknown parameters and $z_{t}$ is a (kx1) vector of deterministic regressors that may include, for instance, a linear time trend in case of $z_{t}=(1, t)^{\prime}$. The regression errors $x_{t}$ are such that

$$
\rho(L ; \theta) x_{t}=u_{t}
$$

where $\rho$ is a function of $L$ and the (px 1$)$ vector $\theta$ and $u_{t}$ is $I(0)$. Robinson (1994) specifies $\rho$ as:

$$
\rho(L ; \theta)=(1-L)^{d_{1}+\theta_{1}}(1+L)^{d_{2}+\theta_{2}} \prod_{j=3}^{p}\left(1-2 \cos w_{r} L+L^{2}\right)^{d_{j}+\theta_{j}},
$$

for given real numbers $d_{1}, d_{2}, \ldots, d_{p}$ and $w_{r}$. Under the null hypothesis:

$$
H_{o}: \theta=0
$$

the above function becomes:

$$
\rho(L)=(1-L)^{d_{1}}(1+L)^{d_{2}} \prod_{j=3}^{p}\left(1-2 \cos w_{r} L+L^{2}\right)^{d_{j}},
$$

and thus, a wide range of possibilities can be tested under $H_{o}(5)$. Some special cases of interest are: 
1. The $I(1)$ model, if $d_{1}=1$ and $d_{j}=0$ for all $j \neq 1$, and in general, $I(d)$ processes for any real $d$ if $d_{1}=d$ and $d_{j}=0$ for all $j \neq 1$.

2. The quarterly I(1) model, if for example, $d_{1}=d_{2}=d_{3}=1 ; d_{j}=0$ for all $\mathrm{j}>3$ and $\mathrm{w}_{\mathrm{r}}=$ $\pi / 2$, and similarly, quarterly $\mathrm{I}(\mathrm{d})$ processes if $\mathrm{d}_{1}=\mathrm{d}_{2}=\mathrm{d}_{3}=\mathrm{d}$.

3. The cyclic $I(1)$ model if $d_{3}=1$ and $d_{j}=0$ for all $j \neq 3$ and similarly, fractional cyclic $I(d)$ processes like (1) if $d_{3}=d$.

Robinson (1994) proposes a Lagrange Multiplier (LM) test for testing $\mathrm{H}_{\mathrm{o}}$ (5) against $\mathrm{H}_{\mathrm{a}}: \theta \neq 0$. Specifically, the test statistic is given by:

$$
\hat{R}=\frac{T}{\hat{\sigma}^{4}} \hat{a}^{\prime} \hat{A}^{-1} \hat{a}
$$

where $\mathrm{T}$ is the sample size, and

$$
\begin{gathered}
\hat{a}=\frac{-2 \pi}{T} \sum_{j}^{*} \psi\left(\lambda_{j}\right) g\left(\lambda_{j} ; \hat{\tau}\right)^{-1} I\left(\lambda_{j}\right) ; \quad \hat{\sigma}^{2}=\frac{2 \pi}{T} \sum_{j}^{*} g\left(\lambda_{j} ; \hat{\tau}\right)^{-1} I\left(\lambda_{j}\right), \\
\hat{A}=\frac{2}{T}\left(\sum_{j}^{*} \psi\left(\lambda_{j}\right) \psi\left(\lambda_{j}\right)^{\prime}-\sum_{j}^{*} \psi\left(\lambda_{j}\right) \hat{\varepsilon}\left(\lambda_{j}\right)^{\prime} \times\left(\sum_{j}^{*} \hat{\varepsilon}\left(\lambda_{j}\right) \hat{\varepsilon}\left(\lambda_{j}\right)^{\prime}\right)^{-1} \times \sum_{j}^{*} \hat{\varepsilon}\left(\lambda_{j}\right) \psi\left(\lambda_{j}\right)^{\prime}\right) \\
\psi\left(\lambda_{j}\right)=\operatorname{Re}\left[\frac{\partial}{\partial \theta} \log \rho\left(e^{i \lambda_{j}} ; \theta\right)\right] \quad \hat{\varepsilon}\left(\lambda_{j}\right)=\frac{\partial}{\partial \tau} \log g\left(\lambda_{j} ; \hat{\tau}\right) ; \quad \lambda_{j}=\frac{2 \pi j}{T},
\end{gathered}
$$

where the function $\mathrm{g}$ above is a known function coming from the spectral density function of $u_{t}$,

$$
f(\lambda ; \tau)=\frac{\sigma^{2}}{2 \pi} g(\lambda ; \tau), \quad-\pi<\lambda \leq \pi
$$

evaluated at $\hat{\tau}=\arg \min { }_{\tau \in T} \sigma^{2}(\tau)$, and $\mathrm{I}\left(\lambda_{\mathrm{j}}\right)$ is the periodogram of $\hat{u}_{t}$, where

$$
\hat{u}_{t}=\rho(L) y_{t}-\hat{\beta}^{\prime} w_{t} ; \quad w_{t}=\rho(L) z_{t} ; \quad \hat{\beta}=\left(\sum_{t=1}^{T} w_{t} w_{t}{ }^{\prime}\right)^{-1} \sum_{t=1}^{T} w_{t} \rho(L) y_{t}
$$


and the summation on $*$ in the above expressions are over $\lambda \in M$ where $M=\{\lambda:-\pi<\lambda<$ $\left.\pi, \lambda \notin\left(\rho_{1}-\lambda_{1}, \rho_{1}+\lambda_{1}\right), 1=1,2, \ldots, s\right\}$, such that $\rho_{1}, 1=1,2, \ldots, \mathrm{s}<\infty$ are the distinct poles of $\psi(\lambda)$ on $(-\pi, \pi]$

Based on the null hypothesis (5), Robinson (1994) showed that, under regularity conditions,

$$
\hat{R} \rightarrow_{d} \quad \chi_{p}^{2} \quad \text { as } \quad T \rightarrow \infty
$$

Thus, a test of (5) against $\mathrm{H}_{\mathrm{a}}: \theta \neq 0$ is given by the rule: "Reject $\mathrm{H}_{\mathrm{o}}(5)$ if $\hat{R}>\chi_{p, \alpha}^{2}$ ", where Prob $\left(\chi^{2}>\chi_{p, \alpha}^{2}\right)=\alpha$. He also showed that the tests are efficient in the Pitman sense that, against local alternatives of form $\mathrm{H}_{\mathrm{a}}: \theta=\delta \mathrm{T}^{-1 / 2}$ for $\delta \neq 0, \hat{R}$ has a $\chi_{p}^{2}(v)$ limit distribution, with a non-centrality parameter $v$ which is optimal under Gaussianity of $u_{t}$.

In the context of the present paper, based on cyclical models, we can particularize the above tests and consider the case with $d_{3}=d$ and $d_{j}=0$ for all $j \neq 3$. Thus, the functions in (4) and (6) become respectively:

$$
\rho(L ; \theta)=\left(1-2 \cos w_{r} L+L^{2}\right)^{d+\theta},
$$

and

$$
\rho(L)=\left(1-2 \cos w_{r} L+L^{2}\right)^{d},
$$

for a given real number $\mathrm{d} ; \mathrm{w}_{\mathrm{r}}=2 \pi \mathrm{r} / \mathrm{T}$ and $\mathrm{r}=\mathrm{T} / \mathrm{s}, \mathrm{s}$ thus indicating the number of periods per cycle. Furthermore, if we impose white noise $u_{t}$, the test statistic greatly simplifies and becomes

$$
\widetilde{R}=\frac{T}{\widetilde{\sigma}^{4}} \frac{\widetilde{a}^{2}}{\widetilde{A}},
$$

where

$$
\widetilde{a}=\frac{-2 \pi}{T} \sum_{j}^{*} \psi\left(\lambda_{j}\right) I\left(\lambda_{j}\right) ; \quad \tilde{\sigma}^{2}=\frac{2 \pi}{T} \sum_{j}^{*} I\left(\lambda_{j}\right)
$$




$$
\tilde{A}=\frac{2}{T} \sum_{j}^{*} \psi\left(\lambda_{j}\right)^{2} ; \quad \psi\left(\lambda_{j}\right)=\log \left|\cos \lambda_{j}-\cos w_{r}\right|
$$

where $\mathrm{I}\left(\lambda_{\mathrm{j}}\right)$ is again the periodogram of $\hat{u}_{t}$ as previously defined. Clearly, a test of (5) against $\mathrm{H}_{\mathrm{a}}: \theta \neq 0$ will have a $\chi_{1}^{2}$ asymptotic distribution.

The tests of Robinson (1994) have been applied to several macroeconomic time series in various articles. Gil-Alana and Robinson (1997) use a version of his tests to test I(d) statistical processes in US historical annual macroeconomic time series. Other versions of the tests, based on monthly and quarterly data, have been respectively studied in Gil-Alana (1999) and Gil-Alana and Robinson (2001), and cyclical models with the tests of Robinson (1994) were also analysed in Gil-Alana (2001). In the following section, we propose a joint test based on Robinson's (1994) procedure for testing simultaneously the need of a linear time trend and the order of integration of the cyclical component in a given raw time series.

\section{A JOINT TEST OF THE TIME TREND AND THE INTEGRATION ORDER}

In Gil-Alana and Robinson (1997), a joint test was proposed for testing simultaneously the need of a linear time trend and the order of integration in a given series at the zero frequency. In this section, a similar test is proposed but, instead of looking at the long run or zero frequency, we concentrate on the cyclical component of the series.

We consider a model given by (2); (3) and (9), with $z_{t}=(1, t)^{\prime}$ for $t \geq 1 ; 0$ otherwise, i.e.,

$$
\begin{gathered}
y_{t}=\alpha+\beta t+x_{t}, \\
\left(1-2 \cos w_{r} L+L^{2}\right)^{d+\theta} x_{t}=u_{t} ; \quad w_{r}=2 \pi r / T,
\end{gathered}
$$

and test the null hypothesis:

$$
H_{o}: \theta=0 \text { and } \beta=0
$$

against the alternative:

$$
H_{a}: \theta \neq 0 \text { or } \beta \neq 0 \text {. }
$$


A joint test is then given by:

$$
\widetilde{S}=\widetilde{R}+\sum_{t=1}^{T} \widetilde{u}_{t} w_{2 t} \times\left(\sum_{t=1}^{T} w_{2 t}^{2}-\frac{\left(\sum_{t=1}^{T} w_{1 t} w_{2 t}\right)^{2}}{\sum_{t=1}^{T} w_{1 t}^{2}} \times \sum_{t=1}^{T} \widetilde{u}_{t} w_{2 t}\right.
$$

with $\mathrm{w}_{\mathrm{t}}=\left(\mathrm{w}_{1 \mathrm{t}}, \mathrm{w}_{2 \mathrm{t}}\right)^{\prime}$ as given above (8), and

$$
\widetilde{u}_{t}=\left(1-2 \cos w_{r} L+L^{2}\right)^{d} y_{t}-\widetilde{\alpha} w_{1 t} ; \quad \widetilde{\alpha}=\left(\sum_{t=1}^{T} w_{1 t}^{2}\right)^{-1} \sum_{t=1}^{T} w_{1 t}\left(1-2 \cos w_{r} L+L^{2}\right)^{d} y_{t},
$$

and $\widetilde{R}$ calculated as described in Section 2 but using the $\widetilde{u}_{t}$ just defined. Then, under $\mathrm{H}_{\mathrm{o}}$ (14), $\widetilde{S} \rightarrow_{d} \chi_{2}^{2}$ as $\mathrm{T} \rightarrow \infty$, and we would compare (16) with the upper tail of the $\chi_{2}^{2}$ distribution. However, in finite samples, the empirical distribution of the tests of Robinson (1994) can vary substantially from the asymptotic results, (see, eg., Gil-Alana, 2000). Thus, in the following section, we report finite-sample critical values of the joint test statistic just described in (16), along with the Robinson's (1994) statistic $\widetilde{R}$ in (11) in the presence of a linear time trend.

\section{A FINITE-SAMPLE EXPERIMENT}

Table 1 reports finite-sample critical values of Robinson's (1994) test statistic $\widetilde{R}$ in (11) in a model given by (12) and (13), i.e., testing the order of integration of the cyclical component in the presence of a deterministic trend, for values of $\mathrm{r}=\mathrm{T} / 2, \mathrm{~T} / 4, \mathrm{~T} / 6, \mathrm{~T} / 7$, $\mathrm{T} / 10$ and $\mathrm{T} / 20 ; \mathrm{T}=40$ and values of $\mathrm{d}=0,0.25,0.50,0.75$ and 1 . We generate Gaussian series obtained by the routines GASDEV and RAN3 of Press, Flannery, Teukolsky and Vetterling (1986) with 50,000 replications in each case, reporting the critical values at the $5 \%$ and the $1 \%$ significance level. We see that for all values of $d$ and $r$, the finite-sample critical values are higher than those given by the $\chi_{1}^{2}$ distribution. Thus, when testing $\mathrm{H}_{\mathrm{o}}(5)$ 
against $\mathrm{H}_{\mathrm{a}}: \theta \neq 0$ with the asymptotic critical values, the tests will reject the null more often than with the finite-sample ones. We also observe across this table slight differences in the values across $d$ and $r$, being higher the differences across $r$, i.e., the number of periods per cycle. However, we do not observe a clear pattern between the critical values and the values of $r$. In general, they are smaller when $r=T / 2$ than when $r=T / 20$, though the critical values do not increase in any case in a monotonic way with respect to $r$.

(Tables 1 and 2 about here)

Table 2 also reports finite-sample critical values but this time based on the joint test $\widetilde{S}$ described in (16), again in a model given by (12) and (13) for the same values of $\mathrm{d}$ and $\mathrm{r}$ as in Table 1 and $\mathrm{T}=40$, which is the sample size used in the empirical application carried out in Section 5. Similarly to Table 1, the finite-sample critical values are higher than those given by the $\chi_{2}^{2}$ distribution, implying that the tests based on these finite-sample values will not reject the null so often as with the asymptotic results of the $\chi_{2}^{2}$ distribution.

We next examine the size and the power properties of the tests in finite samples, comparing the results with those based on the asymptotic results from the $\chi^{2}$ distributions. Tables 3 and 4 report respectively the rejection frequencies of $\widetilde{R}$ in (11) and $\widetilde{S}$ in (16) using both, the finite-sample critical values computed in Tables 1 and 2 and the asymptotic results given by the $\chi_{1}^{2}$ and $\chi_{2}^{2}$ distributions.

Starting with the tests of Robinson (1994), in Table 3, we assume that the true model is given by

$$
\begin{gathered}
y_{t}=1+t+x_{t} \\
\left(1-2 \cos w_{r} L+L^{2}\right) x_{t}=\varepsilon_{t}
\end{gathered}
$$

and look at the rejection frequencies of $\widetilde{R}$ in (11) in a model given by (12) and (13) with d $=1 ; \theta=-1,-0.75,-0.50,-0.25$ and 0 , and the same values of $\mathrm{r}(=\mathrm{T} / 2, \mathrm{~T} / 4, \mathrm{~T} / 6, \mathrm{~T} / 7, \mathrm{~T} / 10$ 
and $\mathrm{T} / 20$ ) under both the null and the alternative hypotheses. Thus, the rejection frequencies corresponding to $\theta=0$ will indicate the sizes of the tests. Looking at the results for the asymptotic tests, we see that the sizes are too large for all values of $r$. Note that a similar problem occurs in Robinson (1994) when using the tests for the long run or zero frequency based on the asymptotic critical values (see, Table 10 in Robinson, 1994). Using, however, the finite-sample critical values, they are smaller but close to the nominal value of $5 \%$. This smaller size of the tests based on the finite-sample critical values is also associated with some inferior rejection frequencies though as we depart from the null, $\theta=$ 0 , throughout $\theta=1$, the difference becomes negligible.

\section{(Tables 3 and 4 about here)}

Table 4 examines the power properties of $\widetilde{S}$ in (16). We assume now that the true model is

$$
\begin{gathered}
y_{t}=1+x_{t} \\
\left(1-2 \cos w_{r} L+L^{2}\right) x_{t}=\varepsilon_{t}
\end{gathered}
$$

and perform $\widetilde{S}$ in (16), testing $\mathrm{H}_{\mathrm{o}}(14)$ in (12) and (13) with $\mathrm{d}=1$ and the same values of $\theta$ and $\mathrm{r}$ as in Table 3. We focus on this particular form of alternatives in order to get better comparisons with Table 3. Similarly to that table, we see that the sizes of the tests based on the asymptotic critical values are too large in all cases while those based on the finitesample ones are smaller and close to the nominal values. Once more, these smaller sizes are also associated with some smaller rejection frequencies in the finite-sample tests, particularly when we are close to the null $\theta=0$. However, we see that for $\theta=0.75$ (and $\theta=$ 1), all the rejection frequencies are higher than 0.900 when using both the finite-sample and the asymptotic critical values. We should finally mention here that increasing the sample size, the difference between the asymptotic and the finite-sample results considerably 
reduces in both tables. However, we only present here the results for $\mathrm{T}=40$ since this is the sample size used in the empirical application in the following section.

\section{AN EMPIRICAL APPLICATION}

We look in this section at the nominal GDP series in the US, the UK, Canada and Japan with annual data from 1960 to 1999. Plots of the original series with their corresponding correlograms and periodograms are given in Figure 1. We observe that all the series increase over the sample period and the nonstationary character of the series seems to assert itself in view of the correlograms (with large and significant values) and the periodograms (with large values around the zero frequency). Figure 2 shows similar pictures for the detrended series (i.e, $\mathrm{x}_{\mathrm{t}}$ in (12) and (13)). We see that the trending behaviour disappears in all cases and the cyclical component becomes apparent in view of the correlograms and the periodograms.

(Figures 1 and 2 about here)

We initially employ $\widetilde{R}$ in (11), testing $\mathrm{H}_{\mathrm{o}}(5)$ in (12) and (13) with values of d equal to 0 , $0.25,0.50,0.75$ and 1 ; white noise $\mathrm{u}_{\mathrm{t}}$, and values of $\mathrm{r}=\mathrm{T} / 2, \mathrm{~T} / 4, \mathrm{~T} / 6, \mathrm{~T} / 7, \mathrm{~T} / 10$ and $\mathrm{T} / 20$, i.e., we test for the order of integration of the cyclical component, including a deterministic trend in the original series. Results are given in Table 5 and we see that $\mathrm{H}_{\mathrm{o}}(5)$ always result rejected for $\mathrm{r}=\mathrm{T} / 2$ and $\mathrm{T} / 4$ (i.e., allowing cycles every 2 or 4 years). However, if the cycles occur every 6 or 7 years, the null cannot be rejected if $d=0.50,0.75$ and 1 for any country. A few more non-rejections appear for the US if $d=0.75$ and $r=T / 20$ and if $d=1$ and $r=$ $\mathrm{T} / 10$; and also if $\mathrm{d}=1$ and $\mathrm{r}=\mathrm{T} / 20$ for the UK and Canada. The non-rejection values obtained when $r=T / 20$ can be due to the fact that the Gegenbauer polynomial becomes similar to $(1-\mathrm{L})$ in case of small frequencies $\mathrm{w}_{\mathrm{r}}$, and thus, for realizations of 40 observations, it can be difficult to distinguish stochastic trends produced by $(1-\mathrm{L})$ and deterministic models. Furthermore, it was shown by Gil-Alana (2001) that if the true model 
contains cycles, for example, every eight periods, the power of Robinson's (1994) tests with $\mathrm{w}_{\mathrm{r}}=\mathrm{T} / 20$ is extremely low. (See, Gil-Alana, 2001, Table 3).

\section{(Tables 5 and 6 about here)}

In Table 6 we employ throughout $\widetilde{S}$ in (16), testing $H_{0}(14)$ in (12) and (13) for the same $(d, r)$ combination as in Table 5, i.e., we test simultaneously for the order of integration and the need of a deterministic trend. We see in this table that if $r=\mathrm{T} / 6$ or $\mathrm{T} / 7$ and $d=0.50,0.75$ or $1, H_{o}(14)$ results now rejected, suggesting in view of the nonrejection values of the previous table that if the cycles occur every six or seven periods, the time trend must be required in these cases. We also observe in this table that the only nonrejection values appear when $d=1$ and $r=T / 4$ for the US, Canada and Japan, while for the UK, $\mathrm{H}_{\mathrm{o}}$ (14) always is rejected, implying the need of a linear time trend at least for this country.

\section{CONCLUDING COMMENTS}

We use in this article a version of the tests of Robinson (1994) for testing the degree of integration of the cyclical component in a given raw time series. A joint test for testing simultaneously the order of integration and the need of a linear time trend has also been proposed. Both tests have standard null and local limit distributions. However, finitesample critical values were computed and several Monte Carlo experiments conducted in the paper showed that the probability of rejection of the true model was much higher under the asymptotic tests than with the finite-sample ones. The tests were applied to annual data of the nominal GDP in the US, the UK, Canada and Japan, the results indicating that a linear time trend is required if the cycles occur every 6 or 7 years, with orders of integration higher than 0.50 and thus, showing the nonstationarity character of the cyclical component of the series. If we do not include a linear trend, the only non-rejected model appears with $d$ 
$=1$ and cycles every 4 years for all countries except the UK, implying the need of the trend if the cycles truly occur every 6 or 7 years and reinforcing its importance in case of the UK.

This article can be extended in several directions. Monte Carlo experiments can be conducted to examine the power of the tests when we misspecify the frequency $\mathrm{w}_{\mathrm{r}}$ of the process in the context of deterministic trends. Note that in the experiments carried out in Tables 3 and 4 we assume the same values for $r$ under both the null and the alternative hypotheses. It would be worthwhile proceeding to get estimates of $\mathrm{w}_{\mathrm{r}}$. However, this would require preliminary differencing to achieve stationarity and invertibility. The tests could have been extended to permit more than one cyclic behaviour in the data and, also to jointly test for a linear time trend and for the order of integration with seasonal fractional integration instead of the cyclical component analysed in this article. Finally, we could also have allowed for weakly parametrically autocorrelated disturbances, computing finitesample critical values and performing the tests on the GDP series. Work in all these directions is now under progress. 


\section{References}

Ahtola, J. and Tiao, G.C. (1987). Distributions of least squares estimators of autoregressive parameters for a process with complex roots on the unit circle. Journal of Time Series Analysis, 8, 1-14.

Bhargava, A. (1986). On the theory of testing for unit roots in observed time series. Review of Economic Studies, 53, 369-384.

Gil-Alana, L.A. (1999). Testing fractional integration with monthly data. Economic Modelling, $16,613-629$.

Gil-Alana, L.A. (2000). Evaluation of Robinson's (1994) tests in finite samples. Journal of Statistical Computation and Simulation 68, 39-64.

Gil-Alana, L.A. (2001). Testing stochastic cycles in macroeconomic time series. Forthcoming in Journal of Time Series Analysis.

Gil-Alana, L.A. and Robinson, P.M. (1997). Testing of unit root and other nonstationary hypotheses in macroeconomic time series. Journal of Econometrics, 80, 241-268.

Gil-Alana, L.A. and Robinson, P.M. (2001). Testing seasonal fractional integration in UK and Japanese consumption and income. Forthcoming in Journal of Applied Econometrics.

Gray, H.L., Zhang. N. and Woodward, W.A. (1989). On generalized fractional processes. Journal of Time Series Analysis, 1, 15-30.

Gray, H.L., Zhang, N. and Woodward, W.A. (1994). On generalized fractional processes. A correction. Journal of Time Series Analysis, 10, 233-257.

Harvey, A. (1985). Trends and cycles in macroeconomic time series. Journal of Business and Economic Statistics, 3, 216-227.

Press, W.H., Flannery, B.P., Teukolsky, S.A. and Wetterling, W.T. (1986). Numerical recipes. The art of scientic computing. Cambridge University Press. Cambridge.

Robinson, P.M. (1994). Efficient tests of nonstationary hypotheses. Journal of the American Statistical Association, 89, 1420-1437. 
Schmidt, P. and Phillips, P.C.B. (1992). LM tests for a unit root in the presence of deterministic trends. Oxford Bulletin of Economics and Statistics, 54, 257-287. 


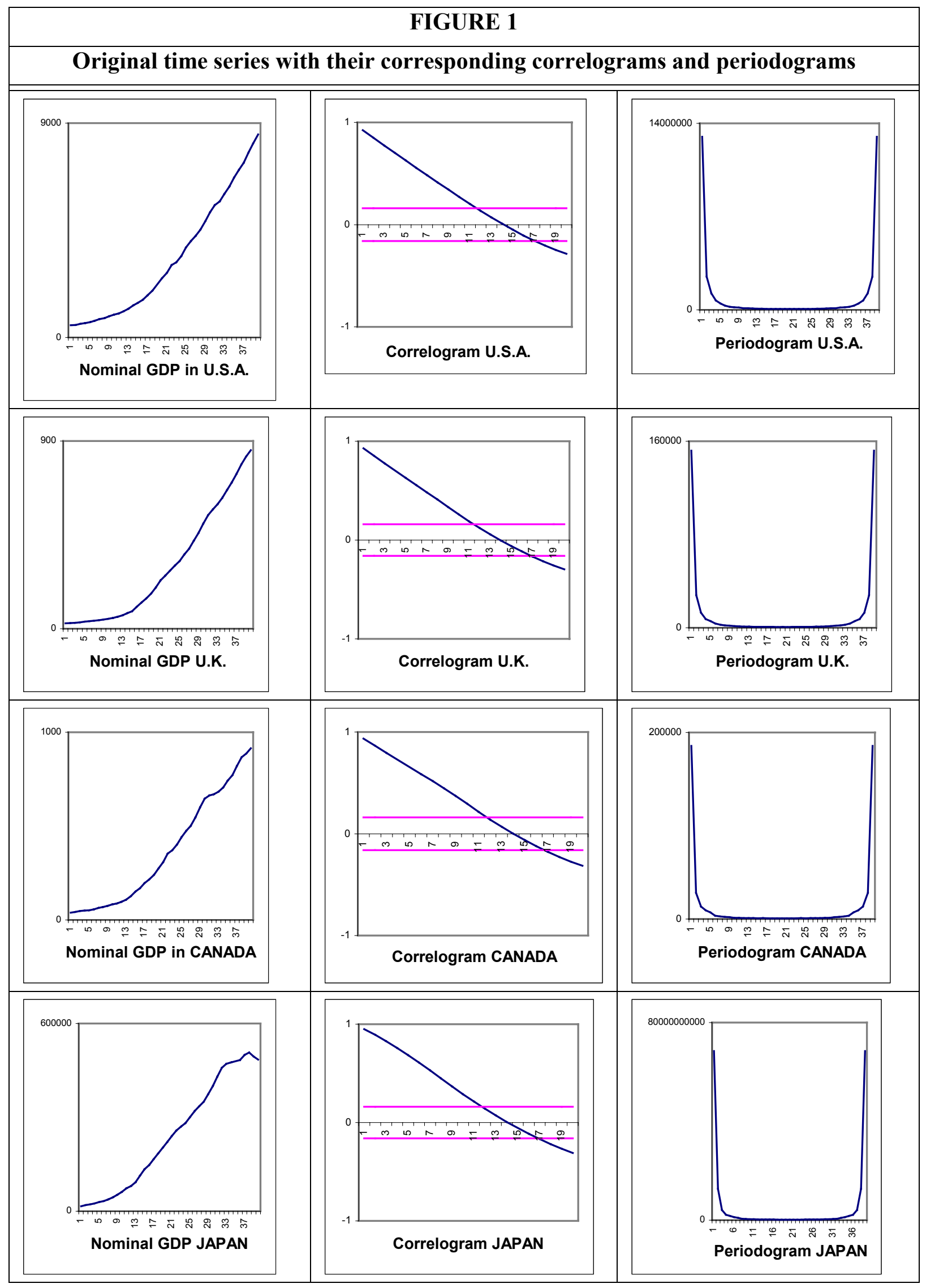




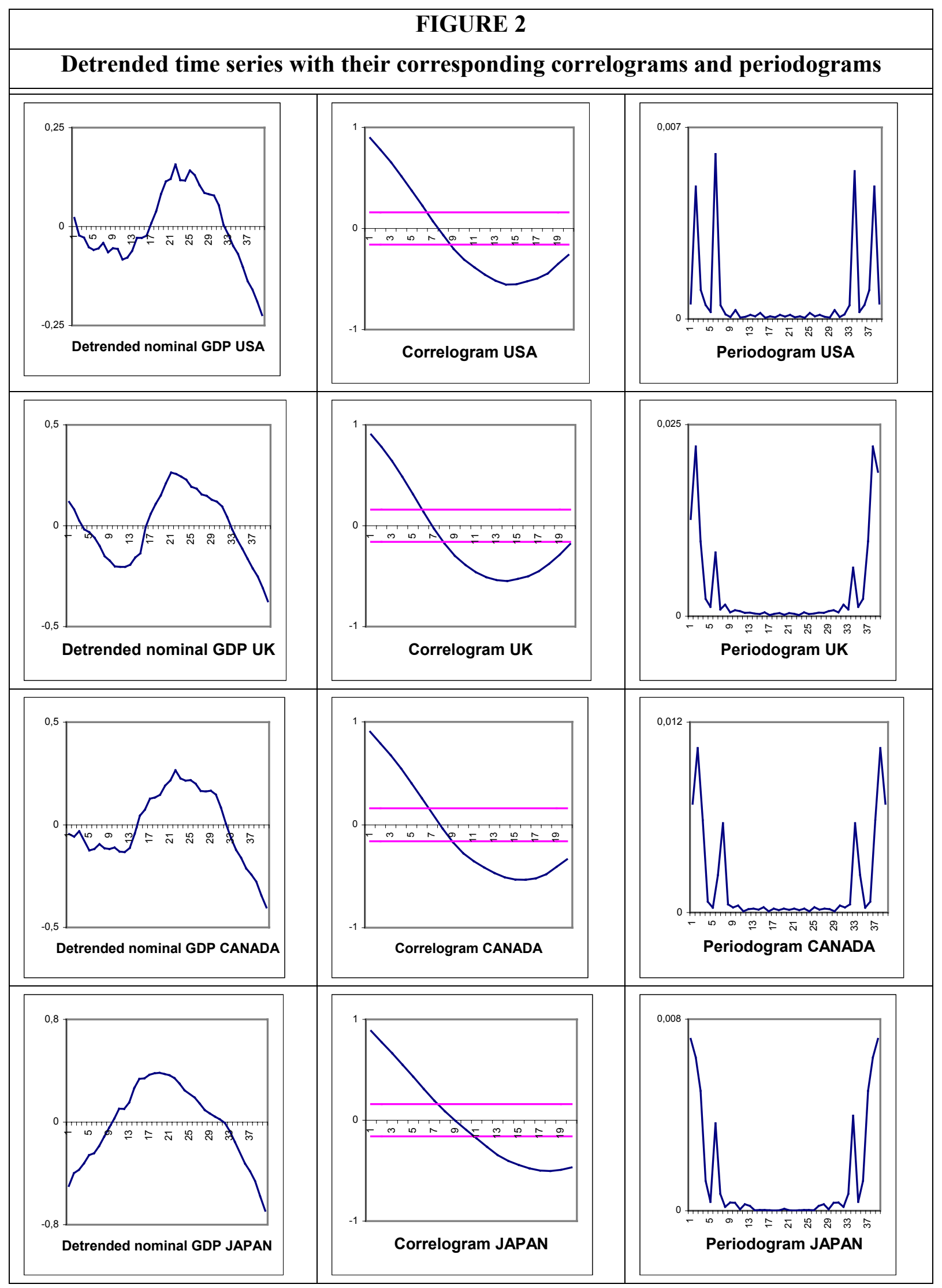




\begin{tabular}{|c|c|c|c|c|c|c|c|c|c|c|c|c|}
\hline \multicolumn{13}{|c|}{ TABLE 1} \\
\hline \multicolumn{13}{|c|}{ Finite-sample critical values of $\widetilde{R}$ in (11) } \\
\hline $\mathrm{T}=40$ & \multicolumn{6}{|c|}{$5 \%$ significance level } & \multicolumn{6}{|c|}{$1 \%$ significance level } \\
\hline $\mathrm{d} / \mathrm{r}$ & $\mathrm{T} / 2$ & $\mathrm{~T} / 4$ & $\mathrm{~T} / 6$ & $\mathrm{~T} / 7$ & $\mathrm{~T} / 10$ & $\mathrm{~T} / 20$ & $\mathrm{~T} / 2$ & $\mathrm{~T} / 4$ & $\mathrm{~T} / 6$ & $\mathrm{~T} / 7$ & $\mathrm{~T} / 10$ & $\mathrm{~T} / 20$ \\
\hline 0 & 4.17 & 6.03 & 6.55 & 6.48 & 6.48 & 7.01 & 6.82 & 8.42 & 9.94 & 9.57 & 9.60 & 9.55 \\
\hline 0.25 & 4.20 & 6.03 & 6.55 & 6.49 & 6.42 & 6.84 & 6.90 & 8.35 & 9.88 & 9.55 & 9.50 & 9.47 \\
\hline 0.50 & 4.18 & 6.02 & 6.52 & 6.45 & 6.30 & 6.54 & 6.90 & 8.31 & 9.85 & 9.55 & 9.31 & 9.11 \\
\hline 0.75 & 4.17 & 6.02 & 6.50 & 6.40 & 6.11 & 6.28 & 6.89 & 8.30 & 9.77 & 9.46 & 9.18 & 8.74 \\
\hline 1.00 & 4.17 & 6.02 & 6.45 & 6.33 & 5.98 & 6.17 & 6.88 & 8.30 & 9.72 & 9.32 & 8.84 & 8.64 \\
\hline
\end{tabular}

In case of $\mathrm{T} / 6$ and $\mathrm{T} / 7, \mathrm{~T}=42$. The critical values of the $\chi_{1}^{2}$ distribution are 3.84 at the $5 \%$ significance level and 6.63 at the $1 \%$ level.

\begin{tabular}{|c|c|c|c|c|c|c|c|c|c|c|c|c|}
\hline \multicolumn{13}{|c|}{ TABLE 2} \\
\hline \multicolumn{13}{|c|}{ Finite-sample critical values of $\widetilde{S}$ in (16) } \\
\hline $\mathrm{T}=40$ & \multicolumn{6}{|c|}{$5 \%$ significance level } & \multicolumn{6}{|c|}{$1 \%$ significance level } \\
\hline $\mathrm{d} / \mathrm{r}$ & $\mathrm{T} / 2$ & $\mathrm{~T} / 4$ & $\mathrm{~T} / 6$ & $\mathrm{~T} / 7$ & $\mathrm{~T} / 10$ & $T / 20$ & $\mathrm{~T} / 2$ & $\mathrm{~T} / 4$ & $\mathrm{~T} / 6$ & $\mathrm{~T} / 7$ & $\mathrm{~T} / 10$ & $\mathrm{~T} / 20$ \\
\hline 0 & 6.50 & 8.31 & 7.85 & 7.60 & 7.37 & 7.50 & 10.31 & 11.71 & 11.08 & 10.58 & 10.15 & 10.16 \\
\hline 0.25 & 6.51 & 8.31 & 7.83 & 7.58 & 7.43 & 7.60 & 10.25 & 11.80 & 11.10 & 10.57 & 10.24 & 10.29 \\
\hline 0.50 & 6.51 & 8.33 & 7.85 & 7.63 & 7.45 & 7.83 & 10.24 & 11.77 & 11.08 & 10.51 & 10.45 & 10.62 \\
\hline 0.75 & 6.50 & 8.33 & 7.85 & 7.63 & 7.53 & 7.93 & 10.32 & 11.84 & 11.08 & 10.51 & 10.59 & 10.93 \\
\hline 1.00 & 6.49 & 8.31 & 7.91 & 7.67 & 7.51 & 7.82 & 10.26 & 11.76 & 11.13 & 10.52 & 10.94 & 10.92 \\
\hline
\end{tabular}

In case of $\mathrm{T} / 6$ and $\mathrm{T} / 7, \mathrm{~T}=42$. The critical values of the $\chi_{2}^{2}$ distribution are 5.99 at the $5 \%$ significance level and 9.21 at the $1 \%$ level. 


\begin{tabular}{|c|c|c|c|c|c|c|c|c|c|c|c|c|}
\hline \multicolumn{13}{|c|}{ TABLE 3} \\
\hline \multicolumn{13}{|c|}{ Rejection frequencies of $\widetilde{R}$ in (11) } \\
\hline \multicolumn{13}{|c|}{ True model: $\mathrm{y}_{\mathrm{t}}=1+\mathrm{t}+\mathrm{x}_{\mathrm{t}} ;\left(1-2 \cos \mathrm{w}_{\mathrm{r}} \mathrm{L}+\mathrm{L}^{2}\right) \mathrm{x}_{\mathrm{t}}=\varepsilon_{\mathrm{t}}$} \\
\hline \multicolumn{13}{|c|}{ Alternative: Testing $\mathrm{H}_{\mathrm{o}}(5)$ in (12) and (13) with $\mathrm{d}=1$} \\
\hline$\theta / \mathrm{r}$ & \multicolumn{2}{|c|}{$\mathrm{T} / 2$} & \multicolumn{2}{|c|}{$\mathrm{T} / 4$} & \multicolumn{2}{|c|}{$\mathrm{T} / 6$} & \multicolumn{2}{|c|}{$\mathrm{T} / 7$} & \multicolumn{2}{|c|}{$\mathrm{T} / 10$} & \multicolumn{2}{|c|}{$\mathrm{T} / 20$} \\
\hline $\mathrm{T}=40$ & FSCV & ASYM & FSCV & ASYM & FSCV & ASYM & FSCV & ASYM & FSCV & ASYM & FSCV & ASYM \\
\hline-1.00 & 0.916 & 0.927 & 0.845 & 0.869 & 0.998 & 0.998 & 0.999 & 0.999 & 0.906 & 0.919 & 0.967 & 0.967 \\
\hline-0.75 & 0.784 & 0.804 & 0.626 & 0.652 & 0.976 & 0.992 & 0.988 & 0.997 & 0.829 & 0.882 & 0.823 & 0.826 \\
\hline-0.50 & 0.715 & 0.730 & 0.353 & 0.372 & 0.751 & 0.880 & 0.819 & 0.924 & 0.419 & 0.585 & 0.692 & 0.693 \\
\hline-0.25 & 0.622 & 0.638 & 0.088 & 0.158 & 0.122 & 0.268 & 0.154 & 0.324 & 0.117 & 0.226 & 0.230 & 0.360 \\
\hline 0.00 & 0.046 & $\mathbf{0 . 0 5 7}$ & 0.041 & 0.144 & 0.043 & 0.096 & 0.045 & 0.091 & 0.045 & 0.135 & 0.041 & 0.131 \\
\hline
\end{tabular}

FSCV and ASYM means finite-sample and asymptotic critical values. The sizes are in bold and the nominal size is $\mathbf{5 \%}$ in all cases.

\begin{tabular}{|c|c|c|c|c|c|c|c|c|c|c|c|c|}
\hline \multicolumn{13}{|c|}{ TABLE 4} \\
\hline \multicolumn{13}{|c|}{ Rejection frequencies of $\widetilde{S}$ in (16) } \\
\hline \multicolumn{13}{|c|}{ True model: $\mathrm{y}_{\mathrm{t}}=1+\mathrm{x}_{\mathrm{t}} ;\left(1-2 \cos \mathrm{w}_{\mathrm{r}} \mathrm{L}+\mathrm{L}^{2}\right) \mathrm{x}_{\mathrm{t}}=\varepsilon_{\mathrm{t}}$} \\
\hline \multicolumn{13}{|c|}{ Alternative: Testing $\mathrm{H}_{\mathrm{o}}(14)$ in (12) and (13) with $\mathrm{d}=1$} \\
\hline$\theta / \mathrm{r}$ & \multicolumn{2}{|c|}{$\mathrm{T} / 2$} & \multicolumn{2}{|c|}{$\mathrm{T} / 4$} & \multicolumn{2}{|c|}{$\mathrm{T} / 6$} & \multicolumn{2}{|c|}{$\mathrm{T} / 7$} & \multicolumn{2}{|c|}{$\mathrm{T} / 10$} & \multicolumn{2}{|c|}{$\mathrm{T} / 20$} \\
\hline $\mathrm{T}=40$ & FSCV & ASYM & FSCV & ASYM & FSCV & ASYM & FSCV & ASYM & FSCV & ASYM & FSCV & ASYM \\
\hline-1.00 & 0.990 & 0.992 & 0.904 & 0.920 & 0.990 & 0.990 & 0.999 & 1.000 & 1.000 & 1.000 & 0.952 & 0.966 \\
\hline-0.75 & 0.901 & 0.913 & 0.901 & 0.903 & 0.980 & 0.985 & 0.988 & 0.993 & 0.999 & 0.999 & 0.902 & 0.911 \\
\hline-0.50 & 0.674 & 0.694 & 0.415 & 0.416 & 0.463 & 0.468 & 0.824 & 0.887 & 0.964 & 0.978 & 0.738 & 0.803 \\
\hline-0.25 & 0.529 & 0.549 & 0.165 & 0.220 & 0.156 & 0.203 & 0.171 & 0.268 & 0.375 & 0.478 & 0.334 & 0.437 \\
\hline 0.00 & 0.049 & 0.061 & 0.047 & 0.124 & 0.049 & 0.116 & 0.047 & 0.067 & 0.044 & 0.056 & \begin{tabular}{|c|}
0.048 \\
\end{tabular} & 0.057 \\
\hline
\end{tabular}

FSCV and ASYM means finite-sample and asymptotic critical values. The sizes are in bold and the nominal size is $5 \%$ in all cases. 
TABLE 5

Testing $\mathrm{H}_{\mathrm{o}}(5)$ in (12) and (13) with $\widetilde{R}$ in (11)

Country: U.S.A.

\begin{tabular}{|c|c|c|c|c|c|c|}
\hline $\mathrm{d} / \mathrm{r}$ & $\mathrm{T} / 2$ & $\mathrm{~T} / 4$ & $\mathrm{~T} / 6$ & $\mathrm{~T} / 7$ & $\mathrm{~T} / 10$ & $\mathrm{~T} / 20$ \\
\hline 0.00 & 18.03 & 23.04 & 21.12 & 21.69 & 18.33 & 53.88 \\
\hline 0.25 & 18.46 & 23.09 & 19.09 & 12.50 & 17.04 & 9.437 \\
\hline 0.50 & 18.64 & 22.95 & 0.07 & $2.26^{\prime}$ & 14.50 & 26.06 \\
\hline 0.75 & 18.72 & 22.74 & 0.05 & $1.90^{\prime}$ & 9.73 & 0.53' \\
\hline 1.00 & 18.75 & 22.51 & 0.02' & $1.34{ }^{\prime}$ & 3.21 ' & 6.47 \\
\hline \multicolumn{7}{|c|}{ Country: U.K. } \\
\hline $\mathrm{d} / \mathrm{r}$ & $\mathrm{T} / 2$ & $\mathrm{~T} / 4$ & $\mathrm{~T} / 6$ & $\mathrm{~T} / 7$ & $\mathrm{~T} / 10$ & $\mathrm{~T} / 20$ \\
\hline 0.00 & 17.97 & 21.06 & 21.23 & 21.16 & 20.47 & 42.41 \\
\hline 0.25 & 18.50 & 21.50 & 9.19 & 9.93 & 18.58 & 47.26 \\
\hline 0.50 & 18.77 & 21.85 & $0.14^{\prime}$ & 2.66 & 16.06 & 41.25 \\
\hline 0.75 & 18.92 & 22.11 & $0.11^{\prime}$ & 2.30 & 12.89 & 14.47 \\
\hline 1.00 & 19.01 & 22.28 & $0.06{ }^{\prime}$ & 1.83 & 8.98 & 0.90 ' \\
\hline \multicolumn{7}{|c|}{ Country: Canada } \\
\hline $\mathrm{d} / \mathrm{r}$ & $\mathrm{T} / 2$ & $\mathrm{~T} / 4$ & $\mathrm{~T} / 6$ & $\mathrm{~T} / 7$ & $\mathrm{~T} / 10$ & $\mathrm{~T} / 20$ \\
\hline 0.00 & 19.32 & 24.41 & 20.12 & 22.93 & 22.60 & 69.46 \\
\hline 0.25 & 19.19 & 24.26 & 15.12 & 19.94 & 20.02 & 64.32 \\
\hline 0.50 & 19.10 & 24.09 & 0.13 & 2.94' & 19.01 & 40.61 \\
\hline 0.75 & 19.05 & 23.91 & 014' & 2.88' & 16.48 & 6.86 \\
\hline 1.00 & 19.02 & 23.72 & 0.15 & 2.71 ' & 10.86 & 1.68 ' \\
\hline \multicolumn{7}{|c|}{ Country: Japan } \\
\hline $\mathrm{d} / \mathrm{r}$ & $\mathrm{T} / 2$ & $\mathrm{~T} / 4$ & $\mathrm{~T} / 6$ & $\mathrm{~T} / 7$ & $\mathrm{~T} / 10$ & $\mathrm{~T} / 20$ \\
\hline 0.00 & 20.80 & 27.35 & 30.03 & 32.55 & 20.81 & 92.32 \\
\hline 0.25 & 20.34 & 27.12 & 25.04 & 22.58 & 19.50 & 77.29 \\
\hline 0.50 & 19.85 & 26.66 & $0.04^{\prime}$ & 2.39' & 15.79 & 48.31 \\
\hline 0.75 & 19.57 & 26.13 & 0.02' & 1.88' & 10.67 & 34.45 \\
\hline 1.00 & 19.41 & 25.56 & 0.03, & 1.13 & 8.02 & 9.35 \\
\hline
\end{tabular}

In bold and ': Non-rejection values at the $95 \%$ significance level. 
TABLE 6

Testing $\mathrm{H}_{\mathrm{o}}$ (14) in (12) and (13) with $\widetilde{S}$ in (16)

Country: U.S.A.

\begin{tabular}{|c|c|c|c|c|c|c|}
\hline $\mathrm{d} / \mathrm{r}$ & $\mathrm{T} / 2$ & $\mathrm{~T} / 4$ & $\mathrm{~T} / 6$ & $\mathrm{~T} / 7$ & $\mathrm{~T} / 10$ & $\mathrm{~T} / 20$ \\
\hline 0.00 & 50.15 & 53.08 & 32.54 & 35.39 & 50.35 & 85.40 \\
\hline 0.25 & 31.66 & 38.08 & 34.13 & 42.78 & 73.79 & 163.67 \\
\hline 0.50 & 26.00 & 24.32 & 37.77 & 54.66 & 118.53 & 480.07 \\
\hline 0.75 & 30.03 & 15.05 & 48.71 & 79.82 & 230.40 & 1872.81 \\
\hline 1.00 & 13.63 & $\mathbf{6 . 0 4}$ & 74.29 & 133.79 & 534.99 & 7848.18 \\
\hline
\end{tabular}

Country: U.K.

\begin{tabular}{|c|c|c|c|c|c|c|}
\hline $\mathrm{d} / \mathrm{r}$ & $\mathrm{T} / 2$ & $\mathrm{~T} / 4$ & $\mathrm{~T} / 6$ & $\mathrm{~T} / 7$ & $\mathrm{~T} / 10$ & $\mathrm{~T} / 20$ \\
\hline 0.00 & 72.95 & 76.41 & 55.02 & 57.82 & 72.95 & 112.11 \\
\hline 0.25 & 44.20 & 52.87 & 57.39 & 69.51 & 112.11 & 247.00 \\
\hline 0.50 & 40.31 & 33.70 & 63.04 & 87.69 & 188.06 & 771.55 \\
\hline 0.75 & 38.92 & 21.21 & 80.3 & 125.02 & 372.04 & 2946.56 \\
\hline 1.00 & 14.10 & 20.23 & 123.13 & 206.28 & 857.14 & 11745.89 \\
\hline
\end{tabular}

Country: Canada

\begin{tabular}{|c|c|c|c|c|c|c|}
\hline $\mathrm{d} / \mathrm{r}$ & $\mathrm{T} / 2$ & $\mathrm{~T} / 4$ & $\mathrm{~T} / 6$ & $\mathrm{~T} / 7$ & $\mathrm{~T} / 10$ & $\mathrm{~T} / 20$ \\
\hline 0.00 & 59.91 & 63.08 & 42.13 & 44.92 & 59.80 & 97.41 \\
\hline 0.25 & 3686 & 44.29 & 44.13 & 54.07 & 90.08 & 200.43 \\
\hline 0.50 & 33.16 & 27.97 & 49.14 & 68.22 & 149.78 & 617.47 \\
\hline 0.75 & 36.53 & 16.40 & 65.05 & 96.90 & 302.33 & 2448.86 \\
\hline 1.00 & 19.85 & $\mathbf{2 . 1 6}$ & 103.10 & 158.72 & 723.08 & 10286.64 \\
\hline
\end{tabular}

Country: Japan

\begin{tabular}{|c|c|c|c|c|c|c|}
\hline $\mathrm{d} / \mathrm{r}$ & $\mathrm{T} / 2$ & $\mathrm{~T} / 4$ & $\mathrm{~T} / 6$ & $\mathrm{~T} / 7$ & $\mathrm{~T} / 10$ & $\mathrm{~T} / 20$ \\
\hline 0.00 & 60.78 & 63.85 & 43.37 & 46.24 & 61,29 & 90.24 \\
\hline 0.25 & 36.38 & 44.61 & 46.06 & 56.63 & 93.52 & 200.49 \\
\hline 0.50 & 42.25 & 28.90 & 53.73 & 75.33 & 160.25 & 722.97 \\
\hline 0.75 & 47.71 & 18.24 & 78.99 & 119.99 & 347.29 & 3345.56 \\
\hline 1.00 & 16.77 & $\mathbf{2 . 7 9}$ & 10.01 & 224.75 & 904.30 & 1894.77 \\
\hline
\end{tabular}

In bold and ': Non-rejection values at the $95 \%$ significance level. 